\title{
Expansion and Contraction of Earth and Sun in Gravitational Field
}

\author{
Branko M. Novakovic
}

\begin{abstract}
The expansion and contraction models of the Earth and of the Sun are derived. It is combined with the experimentally observations data. Further, it is shown that at the minimal non-null radiuses (without singularities), the normalized accelerations of the Earth and of the Sun, are positive. On the other hand, at the maximal non-infinity radiuses, the normalized acceleration of the Earth and of the Sun, are negative. Thus, the Earth and the Sun expansions and contractions are cyclic. The maximal expansion and contraction velocities are close to the speed of light, but less than it. The observed amounts of the Earth and of the Sun masses give very small minimal and maximal radiuses. This means that both the Earth and the Sun must contain more masses.

Assuming that maximal radiuses of the Earth and of the Sun are equal to their present radiuses, there are calculated the related new masses: $M_{e}$ new $=5.662411 \cdot 10^{29} \mathrm{~kg}, M_{s}$ new $=6.185902 \cdot 10^{31} \mathrm{~kg}$. The ratios between new and observed masses are very large. This can be explained by the assumption that the Earth and the Sun contain a dark matter. The ratio between observed and calculated Sun masses is about $3 \%$. This corresponds to the observation that our Universe contains about $3 \%$ of the visible matter in the total matter of the Universe. On the other hand, the ratio between observed and calculated Earth masses is about $0.001 \%$. This ratio is an enigma and is in question? The minimal transition time from the minimal radius to the next quantum state for the Earth is about 7 minutes and for the Sun is about 13 hours.
\end{abstract}

Index Terms - Earth, Sun, Maximal and Minimal radiuses, Expansion and Contraction, Dark matter

\section{INTRODUCTION}

Distant future of the Earth and the Sun are discussed in [1]. In that sense the new version of the Rewiers' law of the mass loss is employed to the Sun [2]. A critical test of empirical mass loss formulas is also used [3]. The dark matter decay between phase transitions at the weak scale and search for invisible axon dark matter experiment are discussed in the references [4] and [5]. Further, the evolution of the star formation rate density of galaxies is presented [6]. A retro perspective of the new version of the Reimers' law of mass loss, stelar evolution and nucleosyntheses, theoretical and applied climatology, conditions of life, sky and telescope are presented in [7-14].

In order to analyze expansions and contractions of the Sun and the Earth, the new Relativistic Alpha Field Theory (RAFT) is employed [15-17]. The new cosmological model, based on the RAF theory is presented in [18]. In the reference [19] it is presented the problem of the minimum time transition between quantum state in a gravitational field. The connection between Planck's and gravitational parameters is

Branko Novakovic, FSB - University of Zagreb, Luciceva 5, P.O.B. 509, 10000 Zagreb, Croatia discussed in [20]. Further, different problems of evolution of the solar systems are considered in [21-36].

The new cosmological model based on the RAF theory is here adapted to the expansion and contraction of the Earth and of the Sun. Thus, the expansion processes of the Earth and the Sun are started from the minimal (non-null) radiuses with the maximal (non-infinite) radial energy density. Further, the accelerating expansion is continuing to the point where the acceleration is equal to zero and velocity is maximal and close to the speed of light, but less than it. At this point acceleration is changed into deceleration and the expansion of the Earth and the Sun is continuing, but now with decreasing of velocity. The expansion process of the Earth and of the Sun is ending at the point where expansion velocity is equal to zero.

At this point the Earth and the Sun have maximal (non-infinite) radiuses and minimal (non-zero) radial energy densities. Since the acceleration is still negative, the contraction process can start. This contraction is continuing to the point where the contraction acceleration is equal to zero. At this point the contraction acceleration is changing into deceleration. Further, the velocity is decreasing to the zero at the initial minimal radius with maximal radial energy density. At this point a new cycle of the Earth and of the Sun motions can start. Thus, here it is described the cyclic motion of the Earth and of the Sun,

The analysis of the Earth and of the Sun motions started by the observed masses: $M_{e}=5.97219 \cdot 10^{24} \mathrm{~kg}$, and $M_{s}=2.0 \cdot 10^{30}$ $\mathrm{kg}$. Meanwhile, the application of the observed masses gives very small minimal and maximal radiuses of the Earth and the Sun. For the Earth we obtain $r_{e \text { min }}=2.217522 \cdot 10^{-3} \mathrm{~m}$ and $r_{e \max }=67.195444 m$. For the Sun the situation is the same: $r_{s \min }=7.797467 \cdot 10^{2} \mathrm{~m}$, and $r_{s \max }=2.362792 \cdot 10^{7} \mathrm{~m}$. Following these analyses one can conclude that both the Earth and the Sun must contain more masses. Therefore, for calculation of the new Earth and the Sun masses we assumed that maximal radiuses of the Earth and the Sun are equal to their present radiuses. The consequence of this assumption is the calculation of the new masses. For the planet Earth it is obtained $M_{e}$ new $=5.662411 \cdot 10^{29} \mathrm{~kg}$, and for the Sun we obtained $M_{\text {s new }}=6.185902 \cdot 10^{31} \mathrm{~kg}$.

The ratio between new and observed masses are very large. Thus, the new Earth mass is $10^{5}$ greater than the observed mass. The new Sun mass is 30 times greater than the observed mass. Therefore, one can ask the question: is it the consequence of the possibility that the Earth and the Sun may contain a dark matter? This problem is also discussed in this article.

The minimal transition time from the minimal radius to the next quantum state for the Earth is about seven minutes and for the Sun is about 13 hours. The transition time from the minimal radius to the maximal radius for the Earth is about 
610 years and for the Sun about 1057 years. The related number of cycles from the beginning to the present time, for the Earth is about 3700 thousand times and for the Sun is about 143 times. Further, the ratio between observed and calculated Earth masses is about $0.001 \%$. On the other hand, the ratio between observed and calculated Sun masses is about $3 \%$. While the Sun masses ratio is in accordance with the Universe observation, the Earth masses ratio is in question.

In the process of the Sun expansion there exists solar evolution with 7 phases [2,3]. In the fourth phase of the Sun evolution (the RGB:tip) with the parameters (age 12.17 Gy and the radius 256 times of the present radius) the Sun has only 0.668 of the present mass. Therefore, the previous results of the Sun parameters calculation are corrected by using the influence of the Solar Evolution Model with Mass Loss [1-5].

\section{EARTH AND SUN EXPANSION AND CONTRACTION MODELS}

For derivation of the new expansion and contraction models for the Earth and for the Sun, one can use Relativistic Alpha Field Theory (RAFT) [15-17] and a new cosmological model [18]:

$$
\begin{gathered}
\left(\frac{\dot{\alpha}}{\alpha}\right)^{2}+\frac{\left(1-\kappa^{2}\right) c^{2}}{\alpha^{2}}=\frac{8 \pi G}{3} \rho\left(1-\frac{2 \pi G}{3} \frac{\rho \alpha^{2}}{c^{2}}\right), \\
\frac{\ddot{\alpha}}{\alpha}=-\frac{4 \pi G}{3}\left(\rho+\frac{3 p}{c^{2}}\right)\left(1-\frac{4 \pi G}{3} \frac{\rho \alpha^{2}}{c^{2}}\right) .
\end{gathered}
$$

Here $\alpha(t)$ is the scale factor and $\dot{\alpha}, \ddot{\alpha}$ are the related time derivations. Parameter $\kappa$ is energy conservation constant. This constant is less than one and bigger than zero and is calculated in the reference [19]. The result of this calculation is the value: $\kappa=0.99993392118$. Parameter $\rho \alpha^{2}$ is radial mass density with the dimension $\mathrm{kg} / \mathrm{m}$.

The maximal expansion and contraction velocities are at the point where the acceleration is zero $(\ddot{\alpha}=0)$ :

$$
\ddot{\alpha}=0, \rho \alpha^{2}=\frac{3 c^{2}}{4 \pi G}, \dot{\alpha}_{1}=+\kappa c, \dot{\alpha}_{2}=-\kappa c .
$$

Here $\rho$ is the fluid mass density $\left(\mathrm{kg} / \mathrm{m}^{3}\right)$ and $\rho \alpha^{2}$ is radial mass density $(\mathrm{kg} / \mathrm{m})$. Thus, at the point where the acceleration is zero the radial mass density is constant and equal to: $3 c^{2} / 4 \pi \mathrm{G}$. Furter, from (2) one can conclude that there exist the expansion and contraction processes of the Earth and of the Sun. This is the common property of particles, planets, galaxies and Universe [19].

The minimal and the maximal radiuses of the Earth and of the Sun are at the points where radial mass density is maximal or minimal, respectively:

$$
\left(\rho \alpha^{2}\right)_{\max }=\frac{3 c^{2}}{2 \pi G}\left(1+\kappa^{2}\right),\left(\rho \alpha^{2}\right)_{\min }=\frac{3 c^{2}}{2 \pi G}\left(1-\kappa^{2}\right) .
$$

From (3) we can see that the Earth and the Sun models have two zero velocities. The first one is at the maximal radial density (minimal radius) and the second is at the minimal radial density (maximal radius).

Now, including maximal and minimal mass density from (3) into the acceleration equation (1) we obtain:

$$
\begin{aligned}
& \left(\frac{\ddot{\alpha}}{\alpha}\right)_{1}=\frac{4 \pi G}{3}\left(\rho+\frac{3 p}{c^{2}}\right)\left(1+2 \kappa^{2}\right), \\
& \left(\frac{\ddot{\alpha}}{\alpha}\right)_{2}=-\frac{4 \pi G}{3}\left(\rho+\frac{3 p}{c^{2}}\right)\left(2 \kappa^{2}-1\right),
\end{aligned}
$$

From (4) one can conclude that at the maximal density (minimal radius) the normalized acceleration of the Earth and of the Sun are positive. On the other hand, at the minimal density (maximal radius) the normalized acceleration of the Earth and of the Sun are negative. Thus, the expansions and contractions of the Earth and of the Sun are cyclic. The maximal expansion and contraction velocities of the Earth and of the Sun are given by the relations:

$$
\dot{\mathrm{\alpha}}_{1 \max }=+\kappa c, \quad \dot{\mathrm{\alpha}}_{2 \max }=-\kappa c .
$$

From (5) we can see that the maximal expansion and contraction velocities are constants and are less than speed of the light in vacuum.

The all spherically symmetric particles (bodies) with mass $M$ have maximal and minimal radial densities [19] given here:

$$
\begin{aligned}
& \rho_{\text {rmax }}=\frac{M}{r_{\text {min }}}=\frac{\left(1+\kappa^{2}\right) c^{2}}{G}=2.693182 \cdot 10^{27} \mathrm{~kg} / \mathrm{m}, \\
& \rho_{r \text { min }}=\frac{M}{r_{\text {max }}}=\frac{\left(1-\kappa^{2}\right) c^{2}}{G}=0.888779 \cdot 10^{23} \mathrm{~kg} / \mathrm{m} .
\end{aligned}
$$

This is very important properties valid in the particles (bodies) physics.

\section{EARTH EXPANSION AND CONTRACTION}

Applying (6) to the Earth with observed mass $\mathrm{M}_{\mathrm{e}}$, one obtains the related minimal and maximal radiuses:

$$
\begin{gathered}
M_{e}=5.97219 \cdot 10^{24} \mathrm{~kg}, \\
r_{\text {emin }}=\frac{M_{e}}{\rho_{\text {rmax }}}=2.217522 \cdot 10^{-3} \mathrm{~m}, \\
r_{\text {emax }}=\frac{M_{e}}{\rho_{\text {rmin }}}=67.195444 \mathrm{~m} .
\end{gathered}
$$

From (7) we can see that the both radiuses are extremally small. Therefore, one can suppose that the maximal Earth radius is equal to the present radius of the Earth. Including this radius, we can calculate the related Earth mass:

$$
\begin{gathered}
r_{\text {max }}=r_{\text {present }}=6371 \cdot 10^{3} \mathrm{~m}, \\
M_{\text {enew }}=\rho_{\text {rmin }} r_{\text {max }}=0.888779 \cdot 10^{23} * 6371 \cdot 10^{3}, \\
M_{\text {enew }}=5.662411 \cdot 10^{29} \mathrm{~kg} .
\end{gathered}
$$

Applying the new (estimated) mass of the Earth (8) to the relations for the minimal and maximal radiuses (7), we obtain:

$$
\begin{gathered}
r_{\text {emin }}=\frac{M_{\text {e new }}}{\rho_{r \text { max }}}=2.102509 \cdot 10^{2} \mathrm{~m}, \\
r_{\text {max }}=\frac{M_{\text {enew }}}{\rho_{\text {rmin }}}=6371,033744 \cdot 10^{3} \mathrm{~m} .
\end{gathered}
$$


Further, one can asks the question: what is the cause of the extremally difference between the observable Earth mass (7) and calculated new mass (8)? This can be explained by the assumption that there exists the dark matter in the Earth (see chapter VI).

The minimal transition time from the minimal radius to the next quantum state for the Earth is calculated here [19]:

$$
\begin{gathered}
\left(\tau_{\psi \rightarrow \psi_{\perp}}\right)_{e}=\frac{2 G M_{e_{\text {new }}}}{\rho_{\text {rmax }}}=4.199182 \cdot 10^{2} \mathrm{~s}, \\
\left(\tau_{\psi \rightarrow \psi_{\perp}}\right)_{e}=6.998635 \mathrm{~min} .
\end{gathered}
$$

Including the maximal number $n_{\max }$ of quantum states from the minimal radius to the radius close to twice of minimal radius [19] one obtains the related transition time:

$$
\begin{gathered}
\left(\tau_{\psi \rightarrow \psi_{\perp}}\right)_{e \max }=n_{\max }\left(\tau_{\psi \rightarrow \psi_{\perp}}\right)_{e}, \\
n_{\text {max }}=30,328.788 \\
\left(\tau_{\psi \rightarrow \psi_{\perp}}\right)_{\text {emax }}=14.709283 \text { days. }
\end{gathered}
$$

In order to calculation of the Earth's expansion process from the minimal to the maximal radiuses we have to know the relation between those radiuses:

$$
\begin{gathered}
r_{\text {min }}=\frac{G M}{(1+\kappa)}, r_{\text {max }}=\frac{G M}{(1-\kappa)}, L_{\text {min }}=2 r_{\text {min }}, \\
\frac{r_{\text {max }}}{r_{\text {min }}}=\frac{(1+\kappa)}{(1-\kappa)}=30,265.847623, \\
n_{L}=\frac{r_{\text {max }}}{2 r_{\text {min }}}=15,132.923831
\end{gathered}
$$

Here $L_{\min }$ is the minimal transition length and $n_{L}$ is the number of the quantum states from the minimal to the maximal radius. Thus, the transition time from $L_{\text {min }}$ to the maximal radius can be calculated by the relations:

$$
\begin{aligned}
& \left(\tau_{\psi \rightarrow \psi_{\perp}}\right)_{\text {etot }}=n_{L}\left(\tau_{\psi \rightarrow \psi_{\perp}}\right)_{\text {emax }}, \\
& \left(\tau_{\psi \rightarrow \psi_{\perp}}\right)_{\text {etot }}=609.847833 \text { years. }
\end{aligned}
$$

The number of cycles from the beginning to the present time for planet Earth are given here:

$$
n_{c-e}=\frac{\text { Earth age }}{2\left(\tau_{\psi \rightarrow \psi_{\perp}}\right)_{\text {etot }}} .
$$

The numerical amount of the related number of cycles for the Earth is as follows:

$$
n_{c-e}=\frac{4.543 \cdot 10^{9} \text { years }}{2 \cdot 609.847833 \text { years }}=3.724699 \cdot 10^{6}
$$

The ratio between observed (official) and calculated Earth masses is:

$$
\begin{aligned}
R_{e}=\frac{M_{\text {eoff }} \cdot 100}{M_{\text {enew }}} & =\frac{5.97219 \cdot 10^{24} \cdot 100}{5.662411 \cdot 10^{29}}, \\
R_{s} & =0.001054 \% .
\end{aligned}
$$

This ratio can be explained by assuming that planet Earth contains a lot of dark mass (see chapter VI).

\section{SUN EXPANSION AND CONTRACTION}

Applying (6) to the Sun with observed mass $\mathrm{M}_{\mathrm{s}}$, one obtains the related minimal and maximal radiuses of the Sun:

$$
\begin{gathered}
M_{s}=2.0 \cdot 10^{30} \mathrm{~kg}, \\
r_{\text {min }}=\frac{M_{s}}{\rho_{r \max }}=7.797467 \cdot 10^{2} \mathrm{~m}, \\
r_{\text {max }}=\frac{M_{s}}{\rho_{\text {rmin }}}=2.362792 \cdot 10^{7} \mathrm{~m}, \\
r_{\text {present }}=6.96 \cdot 10^{8} \mathrm{~m} .
\end{gathered}
$$

From (16) we can see that the maximal radius is less than the present Sun radius. This means that the observed (official) Sun mass must be bigger. In order to estimate the new Sun mass, here it is supposed that the maximal Sun radius is equal to the present radius of the Sun. Using this proposition, one can calculate the related new Sun mass:

$$
\begin{gathered}
r_{\text {smax }}=r_{\text {spresent }}=6.96 \cdot 10^{8} \mathrm{~m}, \\
M_{\text {snew }}=\rho_{\text {rmin }} r_{\text {smax }}=0.888779 \cdot 10^{23} * 6.96 \cdot 10^{8}, \\
M_{\text {snew }}=6.185902 \cdot 10^{31} \mathrm{~kg} .
\end{gathered}
$$

Difference between observed (official) Sun mass (16) and mew mass (17) can be explained by the assumption that there exists the dark matter in the Sun (see chapter VI). Now, applying the calculated (estimated) new mass of the Sun (17) to the relations for the minimal and maximal radiuses (16), we obtain:

$$
\begin{gathered}
r_{\text {min }}=\frac{M_{s \text { new }}}{\rho_{\text {rmax }}}=2.296874 \cdot 10^{4} \mathrm{~m}, \\
r_{\text {max }}=\frac{M_{\text {snew }}}{\rho_{\text {rmin }}}=6.96 \cdot 10^{8} \mathrm{~m} .
\end{gathered}
$$

Further, the minimal transition time from the minimal radius to the next quantum state for the Sun is:

$$
\begin{gathered}
\left(\tau_{\psi \rightarrow \psi_{\perp}}\right)_{s}=\frac{2 G M_{s_{\text {new }}}}{\rho_{\text {rmax }}}=4.587396 \cdot 10^{4} s, \\
\left(\tau_{\psi \rightarrow \psi_{\perp}}\right)_{s}=12.742768 \text { hours. }
\end{gathered}
$$


Including the maximal number $n_{\max }$ of quantum states from the minimal radius to the radius close to twice of minimal radius $[15-17,19]$ one obtains the related transition time:

$$
\begin{gathered}
n=\frac{2 L_{\text {min }}-L_{\text {min }}}{L_{d}}, n=1,2, . ., n_{\text {max }}, L_{d}=1, \\
n=n_{\text {max }},\left(\tau_{\psi \rightarrow \psi_{\perp}}\right)_{\text {smax }}=n_{\max }\left(\tau_{\psi \rightarrow \psi_{\perp}}\right)_{s}, \\
\left(\tau_{\psi \rightarrow \psi_{\perp}}\right)_{\text {smax }}=1056.596441 \text { years. }
\end{gathered}
$$

Here $n_{\max }$ is given in (11). In order to calculation of the expansion process from the minimal to the maximal radiuses of the Sun we have to know the relation between those radiuses:

$$
\begin{gathered}
r_{\text {min }}=\frac{G M}{(1+\kappa)}, r_{\text {max }}=\frac{G M}{(1-\kappa)}, L_{\text {min }}=2 r_{\text {min }}, \\
\frac{r_{\text {max }}}{r_{\text {min }}}=\frac{(1+\kappa)}{(1-\kappa)}=30,265.847623, \\
n_{L}=\frac{r_{\text {max }}}{2 r_{\text {min }}}=15,132.923831
\end{gathered}
$$

Thus, the transition time from $L_{\text {min }}$ to the maximal radius of the Sun can be calculated by the relations:

$$
\begin{gathered}
\left(\tau_{\psi \rightarrow \psi_{\perp}}\right)_{\text {stot }}=n_{L}\left(\tau_{\psi \rightarrow \psi_{\perp}}\right)_{\text {smax }}, \\
\left(\tau_{\psi \rightarrow \psi_{\perp}}\right)_{\text {stot }}=15.989393 \cdot 10^{6} \text { years. }
\end{gathered}
$$

The number of cycles from the beginning to the present time of the Sun is given here:

$$
\begin{gathered}
n_{c-s}=\frac{\text { Sun age }}{2\left(\tau_{\psi \rightarrow \psi_{\perp}}\right)_{\text {stot }}}, \\
n_{c-s}=\frac{4.57 \cdot 10^{9} \text { yars }}{2 \cdot 15.989393 \cdot 10^{6} \text { yars }}=142.9 \approx 143 .
\end{gathered}
$$

The mass ratio between observed (official) and calculated new mass is:

$$
\begin{gathered}
R_{s}=\frac{M_{\text {soff }} \cdot 100}{M_{\text {snew }}}=\frac{2 \cdot 10^{30} \cdot 10 \mathrm{~kg}}{6.185902 \cdot 10^{31} \mathrm{~kg}}, \\
R_{s}=3.233158 \% .
\end{gathered}
$$

Compare the relations in (23a) with the related relations of the planet Earth (15a) one can conclude that the Sun contains less dark mater than planet Earth (see chapter VI). But, (23a) is in accordance with the estimation in the Universe.

\section{Influence Of the SOlar Evolution Model With Mass LOSS}

In the process of the Sun expansion there exists solar evolution with 7 phases $[2,3]$. These phases are compared with the present values of the Sun parameters: age $4.58 \mathrm{~Gy}$, mass $2.0 \cdot 10^{30} \mathrm{~kg}$, radius $6.96 \cdot 10^{8} \mathrm{~m}$, luminosity 1 and effective temperature $5774 \mathrm{~K}$.

The first phase of the Sun evolution started on the zero-age main sequence (ZAMS) with the parameters: the zero age, mass equal to the present mass, radius equal to the present radius, luminosity about $70 \%$ of the current value and the effective temperature $5596 \mathrm{~K}$.

The second phase is MS: hottest one with the parameters: age $7.13 \mathrm{~Gy}$, mass equal to the present mass, radius 1.26 of the present radius, luminosity about 1,11 of the current value and the effective temperature $5820 \mathrm{~K}$.

The third phase is MS: final with parameters: age 10,00 Gy, mass equal to the present mass, radius 1.37 of the present radius, luminosity 1.84 of the current value and the effective temperature $5751 \mathrm{~K}$.

The fourth phase is RGB:tip with the parameters: age 12.17 Gy, mass 0.668 of the present mass, radius 256 of the present radius, luminosity 2730 of the current value and the effective temperature $2602 \mathrm{~K}$.

The fifth phase is ZA-He with the parameters: age $12.17 \mathrm{~Gy}$, mass 0.668 of the present mass, radius 11.2 of the present radius, luminosity 53.7 of the current value and the effective temperature $4667 \mathrm{~K}$.

The sixth phase is $A G B$ : tip with the parameters: age 12.30 Gy, mass 0.546 of the present mass, radius 149 times of the present radius, luminosity 2090 of the current value and the effective temperature $3200 \mathrm{~K}$.

The seventh phase is $A G B$ : tip-TP with the parameters: age $12.30 \mathrm{~Gy}$, mass 0.544 of the present mass, radius 179 times of the present radius, luminosity 41870 of the current value and the effective temperature $3467 \mathrm{~K}$.

The evolution of the Sun contains a mass loss process. In that sense a new mass-loss algorithm is employed by Schröder and Cuntz [2,3]. The important result is that the Sun loss the mass as an RGB giant. This means that as RGB giant the Sun contains only 0.332 of the present Sun's mass. This corresponds to the age of 7.59 Gy from now. As the consequence of the mass loss of the Sun, planet Earth potentially may have a significant orbital expansion because it is the inversely proportional to the remaining solar mass. General Relativity approach are pointed out.

In the reference [1], it is calculated that the Earth will not escape the Sun engulfment. It is because the related hypothetical planets must have the present day minimum orbital radius of about $1.15 \mathrm{AU}$, in order to survive the Sun engulfment. In the same reference it is discussed the chances of finding planets around the Sun as White Dwarf.

Here it is calculated maximal mass of the Sun by using condition that the minimal radial density is at the maximal radius at the $R G B$ - tip phase. Thus, the maximal Sun radius can be obtained from the relation:

$$
\begin{gathered}
r_{\text {smax }}=r_{R G B}=256 \cdot r_{\text {s present }}, \\
r_{\text {smax }}=256 \cdot 6.96 \cdot 10^{8}, \\
r_{\text {smax }}=1.78176 \cdot 10^{11} \mathrm{~m} .
\end{gathered}
$$

The corresponding mass of the Sun follows from the minimal radial density at the maximal radius: 


$$
\begin{gathered}
M_{\text {snew }}=\rho_{\text {rmin }} r_{\text {smax }}, \\
M_{\text {snew }}=0.888779 \cdot 10^{23} * 1.78176 \cdot 10^{11}, \\
M_{\text {snew }}=1.583590871 \cdot 10^{34} \mathrm{~kg} .
\end{gathered}
$$

This result should be corrected by including influence of the mass loss in the RGB: tip phase:

$$
\begin{aligned}
& M_{\text {snew } R G B}=0.668 \cdot M_{\text {snew }}, \\
& M_{\text {snew } R G B}=1.057838 \cdot 10^{34} \mathrm{~kg} .
\end{aligned}
$$

The corrected maximal Sun radius is:

$$
r_{\text {smax cor }}=1.190215 \cdot 10^{11} \mathrm{~m} \text {. }
$$

Further, it is discussed the possibility that the Earth will escape the Sun engulfment. In the reference [1], it is calculated that the present day minimum orbital radius of the hypothetical planets should be about $1.15 A U$. In order to prove it, one should take into a count the Sun mass loss. Therefore, the maximal Sun radius will smaller than in the case of the full Sun mass maximal radius. Present distance, $d_{p}$, between Earth and Sun is equal to $1 A U$. The minimal distance between the Sun and the Earth will be when both the Earth and the Sun have the maximal radius. Thus, the condition that the Earth will escape the Sun engulfment is:

$$
\left(\left(r_{\text {smaxcor }}+r_{\text {earth max }}\right)-\left(r_{\text {spres }}+r_{\text {earthpres }}\right)\right)<1 A U \text {. }
$$

The numerical values of the radiuses in (28) are:

$$
\begin{gathered}
r_{s \max R G B}+r_{\text {emax }}=1.190278 \cdot 10^{11} \mathrm{~m}, \\
r_{\text {spres }}+r_{\text {epres }}=7.02371 \cdot 10^{8} \mathrm{~m},
\end{gathered}
$$

The minimal difference between maximal and present radiuses:

$$
\begin{gathered}
\text { delta }=\left(r_{\text {smax } R G B}+r_{\text {emax }}\right)-\left(r_{\text {spres }}+r_{\text {epres }}\right), \\
\text { delta }=1.183255 \cdot 10^{11} \mathrm{~m} .
\end{gathered}
$$

From (29) and (30) we obtain difference between $1 A U$ and the sum of the maximal radiuses of the Sun and of the Earth:

$$
\begin{gathered}
1 A U-\text { delta }=0.312724 \cdot 10^{11} \mathrm{~m}, \\
1 A U-\text { delta }=0.21 A U .
\end{gathered}
$$

Thus, if (31) is correct than one can conclude that the planet Earth will escape the Sun engulfment.

\section{Do EARTH AND SUn CONTAIN DARK MATER?}

In order to explain difference between observed (official) and calculated mass of the Earth $(7,8)$, we started with calculation of the related ratio, $R_{e}$ :

$$
\begin{gathered}
M_{e}=5.97219 \cdot 10^{24}, \\
M_{\text {enew }}=5.662411 \cdot 10^{29} \mathrm{~kg}, R_{e}=\frac{M_{e} \cdot 100}{M_{\text {enew }}}, \\
R_{e}=\frac{5.97219 \cdot 10^{24} \cdot 100}{5.662411 \cdot 10^{29}}=0.0010547 \% .
\end{gathered}
$$

As we can see from (32), the ratio between observed (official) and calculated Earth masses is very small, compare to the observation that our Universe contains about $3 \%$ of the visible matter in the total matter of the Universe. It can be expected that the new observation of the dark matter, including the Earth's dark matter, could solve these problem [37-40]. Further possibility is that the observation of the Earth mass is no correct?

Now, it is calculated the ratio $R_{S}$ between observed and calculated mass of the Sun $(16,17)$ :

$$
\begin{gathered}
M_{s}=2.0 \cdot 10^{30} \mathrm{~kg}, \\
M_{\text {snew }}=6.185902 \cdot 10^{31} \mathrm{~kg}, R_{s}=\frac{M_{s} \cdot 100}{M_{\text {snew }}}, \text { (33) } \\
R_{s}=\frac{2.0 \cdot 10^{30} \cdot 100}{6.185902 \cdot 10^{31}}=3.233158 \% .
\end{gathered}
$$

From (33), one can see that the ratio between observed (official) and calculated Sun masses is about 3\%. This corresponds to the observation that our Universe contains about $3 \%$ of the visible matter in the total matter of the Universe. This un-visible matter is called the dark matter $[4,5]$. Therefore, the main question is: do the difference between observed (official) and calculated Sun masses are consequences of the existence of the dark matter?

Now we can continue with the discussion about the dark matter in the Earth and the Sun. Let the total mass is denoted by $M_{T}$ and the observed mass by $M_{o}$. In that case dark mass, $M_{D}$, can be calculated by the relation:

$$
M_{T}=M_{D}+M_{o}, M_{D}=M_{T}-M_{o} .
$$

Following (34), one can calculate the quantity of the dark matter in the Earth:

$$
\begin{aligned}
& M_{D e}=M_{T e}-M_{o e}=M_{\text {enew }}-M_{e}, \\
& M_{D e}=5.662351 \cdot 10^{29} \mathrm{~kg} .
\end{aligned}
$$

The quantity of the dark matter in the Sun is given here:

$$
\begin{gathered}
M_{D s}=M_{T s}-M_{o s}=M_{\text {snew }}-M_{s}, \\
M_{D s}=5.985902 \cdot 10^{31} \mathrm{~kg} .
\end{gathered}
$$

Following the previous calculation (32-36), we can conclude the following:

a) The radial mass density at the characteristic points of the Earth and of the Sun models (the minimal radiuses, the maximal radiuses and the maximal expansion, or contraction velocities) are constants 
and are not functions of the mater, dark matter and dark energy.

b) Since the observable masses of the Earth and of the Sun do not correspond to the calculations of their characteristic pointes, there should be another mass (dark matter) that satisfies the previous calculations.

c) Enigma is with the Earth mass, where the ratio between observed and calculated masses is only $0.0010547 \%$. Maybe the new observation of the dark matter, enclouding the Earth's dark matter, could solve these problem [37-40]. Another possibility is that the observation of the Earth mass is no correct?

Recently explanation for dark matter and dark energy is presented in [41]: a) The formation of neutron groups is what we observe as dark matter. b) The dark energy is the cause of the accelerated expansion of the universe. c) Instead, the difference in recession velocity for more distant galaxies is caused by the gravitational acceleration directed towards the center of the universe.

\section{CONCLUSION}

The process of the expansion and contraction of the Earth's and Sun's models are presented. At the minimal (non-null) radiuses, the accelerations are positive. On the other hand, at the maximal (non-infinity) radiuses, the accelerations are negative. Thus, the Earth and the Sun expansions and contractions are cyclic. The maximal expansion and contraction velocities are close to the speed of light, but less than it. The observed (official) amounts of the Earth and the Sun masses give very small minimal and maximal radiuses. This means that both the Earth and the Sun must contain more masses. The ratio between observed (official) and calculated masses for the Sun is about 3\%. The related ratio for the Earth is about $0.0010547 \%$ and is in question. The previous ratios can be explained by the assumption that the Earth and the Sun contain a dark matter. The minimal transition time from the minimal radius to the next quantum state, and from the minimal radius to the maximal radius for the Earth and for the Sun are calculated. The calculated Sun parameters are corrected by using the influence of the Solar Evolution Model with Mass Loss.

\section{ACKNOWLEDGMENTS}

This work was supported by the National Scientific Foundation of Republic of Croatia (grant number: 120-1201842-3048).

\section{REFERENCES}

[1] Schröder K. P. and R. C. Smith, Distant future of the Sun and Earth revisited, arXiv:0801.4031v1 [astro-ph] 25 Jan 2008.

[2] Schröder K.P. and Cuntz M., A New Version of Reimers' Law of Mass Loss Based on a Physical Approach. arXiv:astro-ph/0507598v1 26 Joly 2005.

[3] Schröder K.P. and Cuntz M., A critical test of empirical mass loss formulas applied to individual giants and super-giants, Astronomy and Astrophysics, A\&A 465, 593-601 (2007) doi:10.1051/0004-6361:2006663

[4] Baker M. J., and Kopp J. Dark Matter Decay between Phase Transitions at the Weak Scale. Phys. Rev, Lett. 119, 061801, 07.
Aug. (2017). DOI: 10.1103/PhysRevLett.119.061801

[5] Du N. et al. Search for Invisible Axon Dark Matter with the Axon Dark Matter Experiment, Phys. Rev. Lett. (2018). doi: 10.1103/PhysRevLett.120.151301

[6] Wirsich. J. The Evolution of Star Formation Rate Density of Galaxies. Amer. Jour. of Modern Physics. Vol. 10, No.1, 2021, pp. 1-6. doi:10.11648/j.ajmp.20211001.11

[7] Asplund M., Grevesse N., Sauval A. J., 2005, in Barnes III T. G.,Bash F. N., eds, Cosmic Abundances as Records of Stellar Evolution and Nucleosynthesis, ASP Conf. Ser. 336, Astron. Soc. Pacific, San Francisco, p. 25 (2004, astro-ph/0410214)

[8] Cox P. M., Betts R. A., Collins M., Harris P. P., Huntingford C., Jones C.D., 2004, Theoretical and Applied Climatology, 78,137

[9] Betts R. A., Cox P. M., Collins M., Harris P. P., Huntingford C., Jones C.D., 2004, Theoretical and Applied Climatology, 78,157

[10] Debes J. H., Sigurdsson S., 2002, ApJ, 572, 556

[11] Franck S., von Bloh W., Bounama C., Steffen M., Sch"onberner D., Schellnhuber H.-J., 2002, in Horneck G., Baumstark-Khan C., eds, Astrobiology: The Quest for the Conditions of Life, Springer-Verlag, Berlin, p. 47

[12] Harrison G., Bingham R., Aplin K., Kellett B., Carslaw K., Haigh J., 2007, A\&G, 48, 2.7

[13] Kandel R., Viollier M., 2005, Sp. Sci. Rev., 120, 1

[14] Laughlin G. P., 2007, Sky \& Telescope, June issue, 32

[15] Novakovic B. Relativistic Alpha Field Theory - Part I: Determination of Field Parameters. Inter. Jour. of New Technology and Research (IJNTR) ISSN: 2454-4116, Vol. 1, Iss. 5, Sept. (2015) https://doi.org./10.31871/IJNTR.15.15

[16] Novakovic B. Relativistic Alpha Field Theory - Part II: Does a Gravitational Field Could be Without Singularity? Inter. Jour. of New Technology and Research (IJNTR) ISSN: 2454-4116, Vol.-1, Iss.-5, Sept. (2015) 31. https://doi.org./10.31871/IJNTR.1.5.16

[17] Novakovic B. Relativistic Alpha Field Theory - Part III: Does Gravitational Force Becomes Positive if $(\mathrm{GM} / \mathrm{rc} 2)>1$ ? Inter. Jour. of New Technology and Research (IJNTR) ISSN: 2454-4116, Vol.-1, Iss.-5, Sept. (2015) 39. https://doi.org./IJNTR.1.5.17

[18] Novakovic B. Is Positive Gravitational Force Source of Dark Energy? Inter. Jour. of New Technology and Research (IJNTR) ISSN: 2454-4116, Vol.-1, Iss.-7, Nov. (2015) 7. https://doi.org/10.31871/IJNTR.1.7.5

[19] Novakovic B. Minimum Time Transition Between Quantum States in Gravitational Field. Amer. Jour. of Modern Physics. Vol. 10, No. 2, 2021, pp. 30-35. doi:10.11648/j.ajmp.20211002.12

[20] Novakovic B. Connection between Planck's and Gravitational Parameters. Inter. Jour. of New Technology and Research (IJNTR) ISSN: 2454-4116, Vol.-6, Iss.-12, Dec. (2020) 7. 24

[21] Penev K., Sasselov D., Robinson F., Demarque P., 2007, ApJ, 655, 1166

[22] Bouvier A. and Wadhwa. The age of the solar system redefined by the oldest $\mathrm{Pb}-\mathrm{Pb}$ age of a meteoritic inclusion. Nature Geoscience. 3 (9) (2010).

[23] Gomes R., Levison, Harold F., Tsiganis K, Morbidelli, Alessandro (2005). Origin of the catac Late Heavy Bombardmet period of the terrestrial planets. Nature. 435 (7041: 466-9.

[24] Zabludoff A. (Spring 2003). Lecture 13: The Nebular Theory of the origin of the Solar System. Retrieved 2006-12-27.

[25] Williams J. (2010). The astrophysical environment of the solar birthplace. Contemporary Physics. 51 (5): 381-396. arhXiv:1008.2973.

[26] Greaves S. (2005). Disks Around Stars and the Growth of Planetary Systems. Science. 307 (5706): 68-71.

[27] D’Angelo G., Lubow S. H. (2010). Tree-dimensional 
Disk-Planet Torques in a Locally Isothermal Disk. The Astrophysical Journal.

724 (1):730-747. aeXiv:1009.4148.

[28] Ayliffe B., Bate M.R. (2009). Gas accretion on to planetary cores: tree-dimensional self-gravitating radiation hydrodynamical calculations. Monthly Notices of the Royal Astronomical Society. 393 (1):49-64. arXiv:0811.1259.

[29] Musielek Z.E. 2004, In Stars as Suns: Activity, Evolution and Planets, IAU Symp. 219, ad A.K. Dupree \& A.O. Benz (san Francisco: ASP), 437

[30] Musielek Z.E., \& Rosner, R. 1988, ApJ, 329, 376 Nieuwenhuijzen, H., de Jager, C. 1990, A\&A, 231, 134

[31] Ong K.K., Musielak Z. E., Rosner R., Suess S.T., \& Sulkanen M. N. 1997, ApJ, 474, L143

[32] Piotto G., at al. 2002, A\&A, 391, 945 Musielak Z. E. 2004, in Stars as Suns: Activity, Evolution and Planets, Symp.219, ed. A. K. Dupree \& A. O. Benz (San Francisco: ASP), 437

[33] Musielak Z. E., \& Rosner R. 1988, ApJ, 329, 376

[34] Nieuwenhuijzen H., \& de Jager C. 1990, A\&A, 231, 134

[35] Ong K. K., Musielak Z. E., Rosner R., Suess S. T., \& Sulkanen M. E. 1997, ApJ, 474, L143

[36] Piotto G., at al. 2002, A\&A, 391, 945

[37] Mapping dark matter https://phys.org/news/2017-07-dark_1.html

[38] New theory on the origin of dark matter https://phys.org/news/2017-08-theory-dark.html

[39] Searching for dark matter inside the Earth https://phys.org/news/2021-07-dark-earth.html

[40] Zooming in on dark matter https://phys.org/news/2020-09-dark.html

[41] Richard L. The Explanation for Dark Matter and Dark Energy, International Journal of Physical and Mathematical Sciences Vol.14, No.11, 2020 https://www.academia.edu/44467185/The_Explanation_for _ Dark_Matter_and_Dark_Energy_IJPMS_

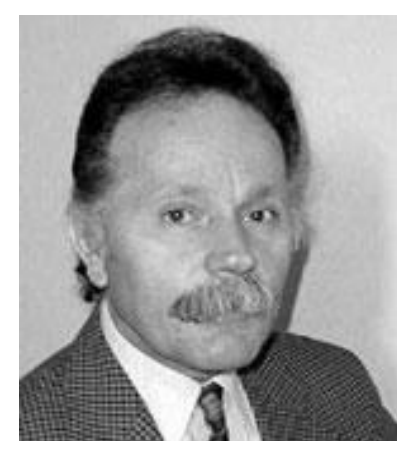

Branko Novakovic is a Professor Emeritus at FSB - University of Zagreb, Croatia. Prof. Novakovic received his $\mathrm{PhD}$ from the University of Zagreb in 1978. His research of interest includes physics, control systems, robotics, neural networks, and fuzzy control. $\mathrm{He}$ is author of three books; Relativistic Alpha Field Theory (RAFT, e-book, 2016), Control Methods in Robotics, Flexible Manufacturing Systems and Processes (1990), Control Systems (1985) and the first author of a book Artificial Neural Networks (1998). He has published over 240 research papers in his research of interest. 